\title{
Peak to Average Power Ratio Reduction in Mc Cdma System by Using Pulse Shaping Technique
}

\author{
Amandeep Singh $^{1}$, Rajbir Kaur ${ }^{2}$, Gagandeep Kaur ${ }^{3}$ \\ ${ }^{1}$ (University college of Engineering, Punjabi University, Patiala, India) \\ ${ }^{2}$ (University college of Engineering, Punjabi University, Patiala, India) \\ ${ }^{3}$ (Guru Nanak Institute of Technology, Mullana, Ambala, India)
}

\begin{abstract}
One of the drawback of multicarrier code division multiple access (MC CDMA) is the high peak to average power ratio (PAPR). In this paper A novel scheme based on pulse shaping described for PAPR reduction. Here three different pulse shaping filters (Raised cosine. Sinc power pulse and Nyqist pulse) are described for PAPR reduction in MC CDMA system. The method is based on the proper selection of the pulses for shaping the different sub carriers of the MC CDMA modulation scheme. It is shown by computer simulation that the scheme achieves the significant improvement in PAPR reduction in MC CDMA system. Its implementation complexity is much low in comparison to the previous published methods. Thus pulse shaping technique can be used not only to shape the spectrum of the transmitted signal but also to reduce its PAPR. In computer simulation It is observed that by using nyquist pulse near about $6.8 \mathrm{db}$ PAPR is obtained. Which is considerable PAPR reduction than a PAPR value of Original MC CDMA signal
\end{abstract}

Keywords: $M C$ CDMA, PAPR, PULSE SHAPING, BPSK

\section{Introduction}

Orthogonal frequency division multiplexing (OFDM) has been adopted for high-speed wireless data transmission systems due to its inherent robustness against multipath fading channels. By combining OFDM and Code Division Multiple access (CDMA), multicarrier code division multiple access (MC-CDMA or OFDMCDMA) inherits the advantages of both OFDM and CDMA techniques. In such systems, the CDMA part provides multiple access ability as well as to spread each user's data across the entire available frequency band The spreading code's chips are then modulated on orthogonal subcarriers and spread across the time domain via the OFDM modulation which provides protection against the delay spread of the multipath channel. Therefore MCCDMA technique can achieve high data rate transmission with protection against both frequency selective fading and time dispersion channel while at the same time offers a spectrum efficient multiple access strategy [1].

Several types of Code Division Multiple Access (CDMA) systems based on the combination of Direct Sequence (DS) CDMA and orthogonal frequency division (OFDM) multiple access techniques are proposed recently. Among them, Multi-Carrier CDMA (MC-CDMA) transmission scheme, characterized by spreading operation in the frequency domain, is one which represents qualitatively new spread spectrum technique, which is dual to DS-CDMA. The MC CDMA transmitter configuration for the $k^{\text {th }}$ user is shown in figure 1.After modulation, user data is fed to the spreader. The nth symbol of the $k^{t h}$ user in the $i^{t h}$ block by user specific spreading code $C_{K}(t)=\left[C_{1}^{K}, C_{2}^{K},----, C_{N c}^{K}\right]$ is the spreading factor of the $k^{t h}$ user is fed to serial to parallel converter and then Inverse Fast Fourier Transform (IFFT) in the frequency domain. MC CDMA uses Inverse Fast Fourier Transform (IFFT) to divide the bandwidth into orthogonal overlapping subcarriers; the nth data symbol for $k^{t h}$ user, $a_{K}(n)$ is spread by user K's corresponding spreading code vector $\mathrm{Ck}$. Each of the $N_{c}$ subcarriers is modulated by a single chip. The data is converted back into serial data before cyclic prefix or guard interval is inserted to combat ISI [2]. Finally the signal is fed to Digital to Analog converter for transmission (DAC) through Rayleigh fading channel with Additive White Gaussian Noise (AWGN).The transmitted signal is represented as

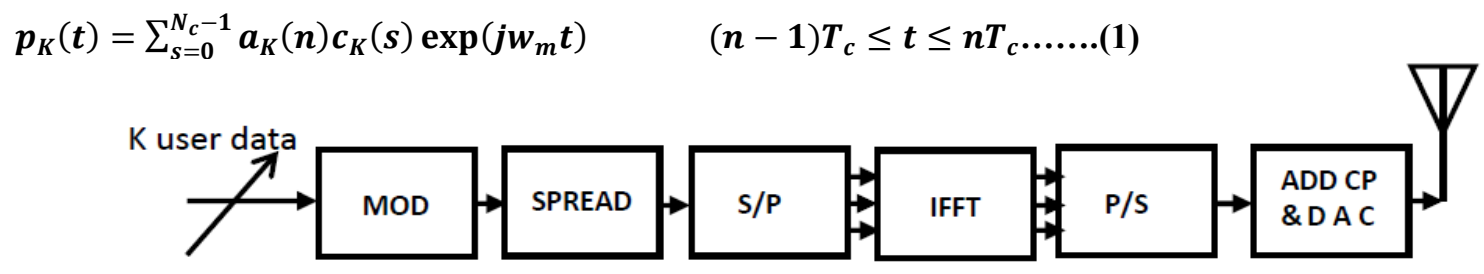

Figure 1. Block Diagram of MC CDMA Transmitter [2] 
MC CDMA is a very attractive technique for high speed data transmission over multipath fading channels. The PAPR problem is one of the most important issues for developing multicarrier transmission systems. MC CDMA is widely used in broadband networks such as Long Term Evaluation (LTE) and broadband communication networks. However, MC CDMA systems have the inherent problem of a high PAPR, which causes poor power efficiency or serious performance degradation in the transmitted signal. This brings disadvantages like complexity of ADC and DAC, reduced power efficiency, and high Bit Error Rate (BER), consumption of more power. High power amplifiers are required which results in increased cost component. Thus, if we reduce PAPR, we shall obtain reduced complexity of Analog to Digital Converter (ADC) and Digital to Analog Converters (DAC), improved signal to noise ratio and Bit Error Rate (BER) [2].

\section{Related work}

To reduce the PAPR, many techniques are proposed. The first one is the signal distortion technique, which introduces distortion to signals and causes degradation in the performance including clipping, windowing, peak cancelling or companding. In companding technique, compression in transmitter and expanding in receiver has been proposed by Wang et al. Clipping is simple and effective and causes In-Band-distortion and increased BER. The companding transforms' performance is better and reduces distortion than to that of the clipping. Another proposal by Yuan Jiang is an algorithm that uses the special airy function and is able to provide an improved Bit Error Rate (BER) and minimized Out of Band Interference (OBI) in order to reduce PAPR effectively [2]. In 1997 author Branislav M. Popovic gives technique for the selection of spreading sequences for the Multi- Carrier CDMA (MC-CDMA) systems with spectrum spreading in the frequency domain. Author have shown that the time domain cross correlation function between the spreading sequences is not a proper interference measure for the asynchronous MC CDMA users. Therefore the spectral correlation function is introduced and, together with the peak-to average power ratio and the dynamic range of the corresponding multicarrier waveforms, is used for the evaluation of MC CDMA sequences. Some well-known classes of sequences, such as Walsh and Gold sequences, as well as Orthogonal Gold and Zadoff-Chu sequences, are evaluated with respect to the aforementioned basic criteria. It is also shown that the multicarrier spread spectrum waveforms based on the (multilevel) Huffman sequences have the lower PAPR than a single sine wave. In the literature, there is a big number of researchers that worked on this problem of PAPR reduction but most of their techniques are categorized as modifying the output signal's amplitude such as clipping and filtering modifying the data's phases/amplitudes and multiple signal representation such as the partial transmit sequence (PTS) and the selective mapping (SLM) and other categories such as the coding [3]. In 2006 scientists Zbynek Fedra and Vladimir Sebesta proposed new PAPR reduction method in MC CDMA system. they introduces spreading sequences among subcarriers. This is used for chip position formatting to PAPR minimization. The optimizations methods are used to get best chip interleaving sequence (namely Genetic Algorithm (GA) and Ant Colony Optimization (ACO)) [5]. In 2007 than other scientists Jizeng Wang, Jingyu Luo and Yanlong Zhang tried to reduce PAPR by a new phase sequence for SLM in MC CDMA system. A new Pseudo Random Interferometry code is proposed as the phase sequence for SLM to reduce PAPR of MCCDMA. the proposed sequence is more effective for PAPR reduction in MC-CDMA system compared with Walsh-Hadamard sequence and Golay sequence [6]. In 2008 scientists Aihua Wang ; Jianping An ; Zhongxia, proposed a method based on immune colonal selection algorithm and hop field neural network. They have shown hybrid solution which adopts both the concept of ICSA and HNN that shows a considerable improvement in PAPR reduction [7]. In 2009 authors Hossein Rahmani and Naaser neda gives a technique based on modified phase approach for reduction of PAPR in MC CDMA system. they consider fading channel as well as the nonlinearity of transmitter's amplifier to show how the control of the PAPR in different approaches can actually affect on the overall quality of the received signal. They have proposed a new method of PAPR reduction in MC CDMA system which is based on phase perturbation after spreading process [8]. In 2010 scientists R.Manjith, S.C.Ramesh, and M.Mohamed Ismail Majeed have described Non linear compandig techniques for PAPR reduction in OFDM and MC CDMA systems. Nonlinear companding technique adjust both large and small signals and can keep the average power at the same level. In addition, the schemes based on nonlinear companding techniques have low implementation complexity and no constraint on modulation format and subcarrier size [9]. In 2011 the scientists Montadar Abas Taher, JS Mandeep, Mahamod Ismail, Hussain Falih Mahdi, Have proposed a novel algorithm to enhance the PAPR of MC CDMA system. The algorithm called Half Length Phase Updating ' (HLPU), it is called half because the phase updating will be applied to only half of the IFFT length $(\mathrm{N} / 2)$. The HLPU will be predefined even one bit of side information from the transmitter to the receiver. This additional block in the system will be inserted before the Inverse Fast Fourier Transform (IFFT) of the Multicarrier Code Division Multiple Access (MC CDMA) system. The simulation results proved that there is $94.2 \%$ reduction in the system complexity and a reduction in the PAPR of around $2 \mathrm{~dB}$ has been obtained [10]. In 2012 authors M. F. Ghanim and M. F. L. Abdullah gives a PAPR reduction method using single carrier FDMA. They presents a novel MCCDMA which has low peak to average power ratio (PAPR). 
The system is designed by combining MC-CDMA with the Single carrier-frequency division multiple access (SC-FDMA) because the later has low PAPR [11]. The very easy and good PAPR performance can be achieved using the amplitude clipping and filtering technique but it is reduces the performance of the BER considerably. The complexity of the PTS makes it not useful in multicarrier systems while the SLM is less complex than the PTS with the same performance of the PAPR and same amount of side information as in the PTS. Another technique is the phase/amplitude weighting. This technique can be considered as a type of the SLM method but it does weighting for the amplitude and or the phase or only the amplitude and or the phase. In this method, still we need to send some side information to the receiver. In the current paper, we have modified the method of but not all its suggested method [3]. It used the weighting as well as the SLM. We will not use the SLM here, but only the weighting process without side information as in with good PAPR performance as compared to the SLM technique.

\section{Papr}

An important parameter that the designer should consider it when designs any multicarrier system, is the PAPR. This can be defined as the maximum peak of the output signal to the

Average power as follows,

$$
\boldsymbol{P A P R}=\frac{\max |s(t)|^{2}}{E\{\mid s(t)\}}
$$

Where $E\{\cdot\}$ denotes the expectation. To get an accurate measure for the PAPR, the signal will be oversampled by a factor of 4 . By this up sampling factor, all the PAPR will be taken. Good way to measure the PAPR is by using its complementary cumulative distribution function (CCDF). The CCDF can be defined as,

$$
\operatorname{CCDF}(P A P R)=\operatorname{Pr}\left(P A P R>P A P R_{0}\right) \ldots \ldots \ldots(2)
$$

\section{The methodology for Papr Reduction}

In this paper a novel technique based on pulse shaping is described to reduce the PAPR with low complexity. It has been shown in literature that PAPR of OFDM can be reduced if the subcarrier waveforms have different shapes [4]. We described that with proper selection of all types pulses to shape the subcarrier waveforms can achieve significant improvement in PAPR reduction. The PAPR performance can be improved by using Nyquist pulse. By making cross correlation close to 1 a multicarrier signal with very low PAPR can be obtained. The cross correlation function is a function of signal modulated symbol and subcarrier waveforms

Note that the cross-correlation function depends on the different time waveforms of the different carriers. Thus a proper selection of the subcarrier time waveforms will increase the cross-correlation function and reduce the PAPR. A set of broadband pulse waveforms that reduces the PAPR was described in literature. They are cyclic shifts of a broadband main shaping pulse. In fact, by observing the properties of Nyquist pulses, we can conclude that all sets which are cyclic shifts of Nyquist pulses whose spectrum is within the OFDM bandwidth can reduce the PAPR. We define the following set of Nyquist pulses:

$$
\begin{aligned}
& S_{n}(t)=0, \quad\left|t-\frac{T}{2}\right|>\frac{T}{2}(n=0,1 \ldots \ldots \ldots . N-1) \ldots \ldots \ldots . .(3) \\
& S_{m}(t)=e^{j 2 \pi m \frac{t}{T}}=S_{i}\left(t-\delta_{m-i)} e^{j 2 \pi \pi_{\bar{T}}^{i}\left(t-\delta_{m-i}\right)}\right. \\
& \text { Where } \boldsymbol{\delta}_{\boldsymbol{m}-\boldsymbol{i})}[(\boldsymbol{m}-\boldsymbol{i}) \boldsymbol{m o d} \boldsymbol{N}] \boldsymbol{T}_{\boldsymbol{s}} \text {. Then PAPR becomes } \\
& P A P R=\frac{1}{N} \max _{0 \leq t \leq T}\left(\sum_{m=0}^{N-1}\left|S_{m}(t)\right|\right)^{2} \leq \frac{1}{N}\left(\max _{0 \leq t \leq T} \sum_{m=0}^{N-1}\left|S_{m}(t)\right|\right)^{2}=N
\end{aligned}
$$

It is concluded that shaping the different subcarrier using the set of Nyquist pulses shape will not increase the peak amplitude of the transmitted signal. The reason is that the peak amplitude of the different waveforms shaped by Nyquist pulse set formed according to equation (5) will not occur at the same time which is also the ISI free property of Nyquist pulse. Note that the highest PAPR is obtained when the sinc power pulse is used. In the following, different pulses are introduced for PAPR reduction. 


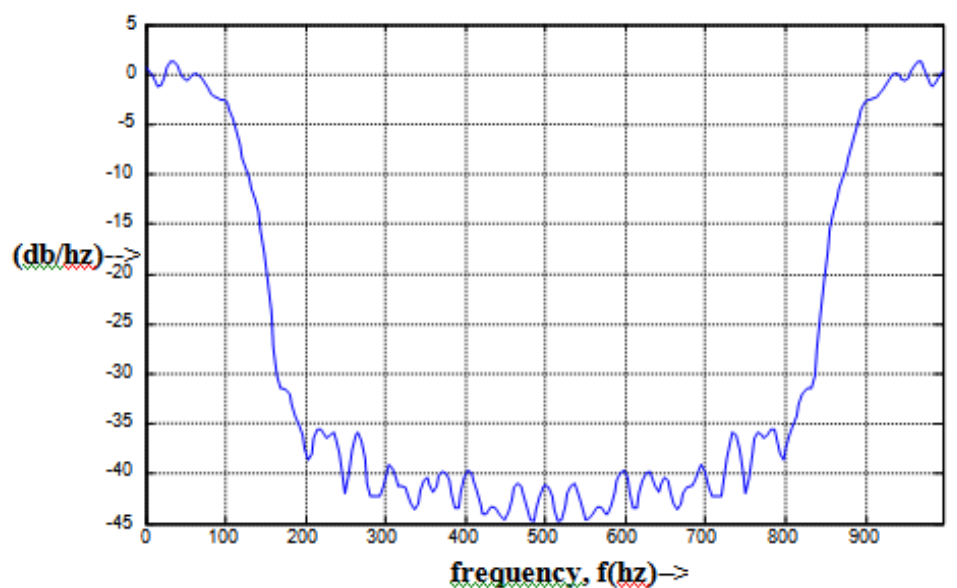

Figure 2 power spectral density with raised cosine filter.

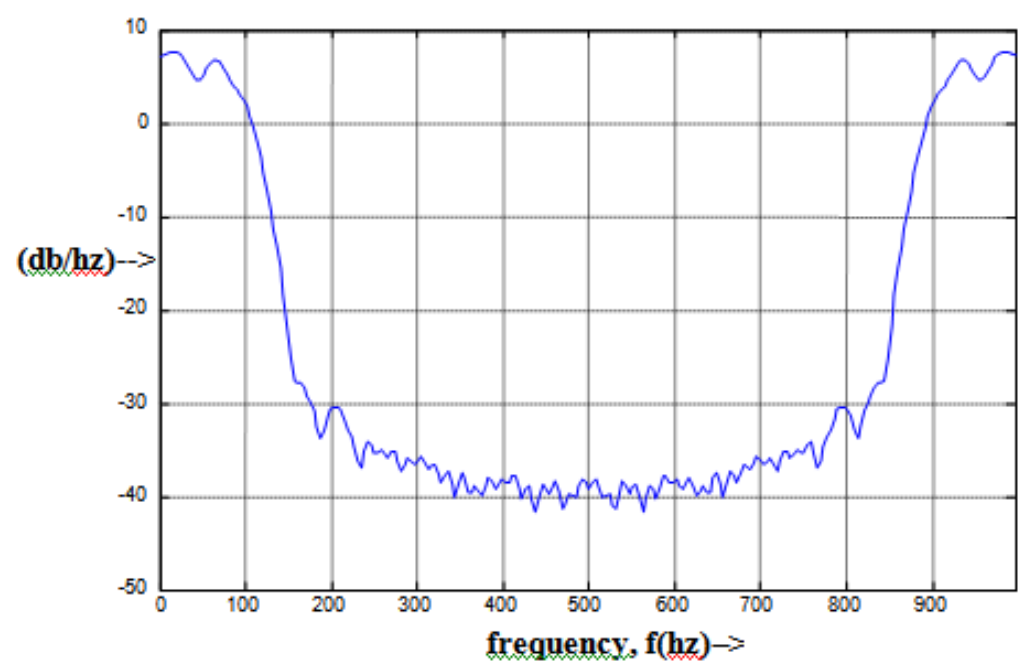

Figure3. power spectral density with Gaussian filter

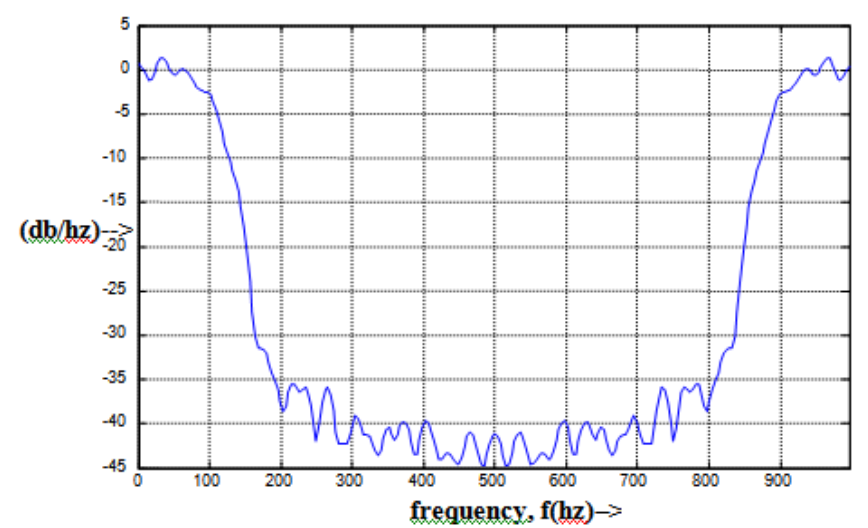

Figure 4 power spectral density with Sin c power filter

Fig.2,3,4 illustrates the power spectral density curves of modulated MC CDMA signal. Frequency response curves comparison of MC CDMA signal have shown above.

\section{Simulation Results}

In simulations we have used three different pulse shaping filters (Raised cosine, Sinc power pulse and nyquist pulse filter) and found Nyquist pulse shaping filter is better than other filters for PAPR reduction in MC CDMA system. Fig 5 illustrates the cumulative distributions function (CCDF) of PAPR of the MC CDMA signals for different pulses. It is observed that our technique provides considerable gain in PAPR reduction as compared to 
previous methods. It is observed that by using nyquist pulse near about $6.8 \mathrm{db}$ PAPR is obtained. It is shown that approximately $.4 \mathrm{db}$ reduction in PAPR from sin c power pulse is obtained by pulse shaping approach.

Main parameters used in simulations
\begin{tabular}{|l|l|}
\hline No. of carriers & 5 \\
\hline Number of bits & 10,000 \\
\hline Modulation scheme & BPSK \\
\hline Oversampling factor & 4 \\
\hline
\end{tabular}

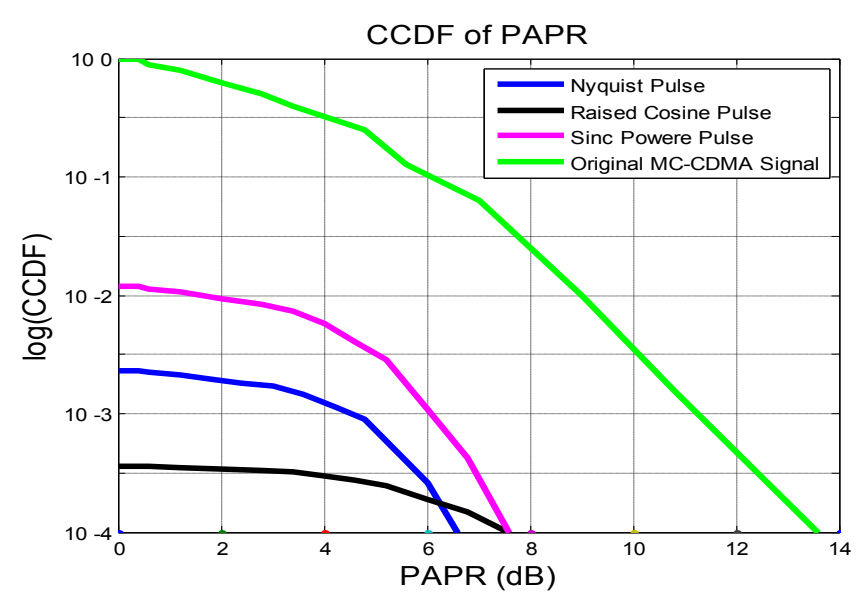

Figure 5 PAPR Comparison by using different pulses

It is shown that by using above approach near about $7 \mathrm{db}$ Reduction in PAPR of original MC CDMA signal is obtained.

\section{Conclusions}

In this paper a pulse shaping based method is described for PAPR reduction in MC CDMA system. The method is based on proper selection of pulses for shaping different subcarriers of MC CDMA scheme.

It is shown by simulation that the given method can achieve significant improvement in PAPR reduction in MC CDMA system. As the better pulse achieves better the performance in PAPR is obtained. The given technique is very effective and flexible for any number of carriers. Although a lot of filter algorithms are already working to reduce the PAPR in any modulation or channel estimation technique. In our approach we have applied three different levels of filter Raised Cosine, Nyquist and Sin c power pulse out of which Nyqusit filter is found a little superior in terms of find the probabilities of PAPR and reducing it.

\section{References}

[1] Lin Yang, Mingli You and Jun Li," Optimized Spreading Code Reallocation Technique for PAPR Reduction in MC-CDMA systems," IEEE conference,pp.1-6, 2009.

[2] B.Sarala and D.S.Venkateswarulu, "MC CDMA PAPR Reduction Techniques Using Discrete Transforms and Companding", International journal of Distributed and Parallel Systems volume 2,NOV. 2011.

[3] Montadar Abas Taher, JS Mandeep, Mahamod Ismail, Hussain Falih Mahdi, "A Novel Simple Algorithm to Enhance the Peak to Average Ratio of MC-CDMA System", IEEE conference, pp.324-326, 2011

[4] Zhang Chen, FU Yaowen, Zhang Eryang, "Peak-To-Average Power Ratio Reduction in OFDM SYSTEM using Nyquist Pulse Shaping Technique", IEEE International Symposium, vol. 2, pp.1522-1525, 2005.

[5] Zbynek Fedra, Vladimir Sebesta, "The new PAPR Reduction approach in MC CDMA", IEEE symposium, pp. 270-273, 2006.

[6] Jizeng Wang, Jingyu Luo, Yanlong Zhang, “A New Phase Sequence for SLM in MC-CDMA System”, IEEE conference, pp. 938 $941,2007$.

[7] Aihua Wang ; Jianping An ; Zhongxia," "PAPR Reduction for MC-CDMA System Based on ICSA and Hopfield Neural Network”, IEEE conference, pp. 5068 - 5071, 2008.

[8] Hossein Rahmani and Naaser neda, "PAPR reduction in MC CDMA signal based on a modified phase approach", IEEE conference, pp. 5068 - 5071, 2009.

[9] R.Manjith, S.C.Ramesh, M.Mohamed Ismail Majeed, "R.Manjith, S.C.Ramesh, and M.Mohamed Ismail Majeed", IEEE conference, pp. $274-278,2010$.

[10] Montadar Abas Taher, JS Mandeep, Mahamod Ismail, Hussain Falih Mahdi, "A novel simple algorithm to enhance the peak to average ratio of MC-CDMA system", IEEE conference, pp. $324-326,2011$.

[11] M. F. Ghanim, M. F. L. Abdullah, "A novel PAPR reduction scheme in MC-CDMA system using Single carrier FDMA technique", IEEE conference, pp. 129 - 134, 2012. 